Check for updates

Cite this: RSC Adv., 2019, 9, 2599

Received 21st November 2018

Accepted 4th January 2019

DOI: $10.1039 / c 8 r a 09598 a$

rsc.li/rsc-advances

\section{Electrochemical impacts of sheet-like hafnium phosphide and hafnium disulfide catalysts bonded with reduced graphene oxide sheets for bifunctional oxygen reactions in alkaline electrolytes $\uparrow$}

\author{
Mayilvel Dinesh Meganathan, (D) ${ }^{a}$ Taizhong Huang, (D) *a Hengyi Fang, ${ }^{a}$ \\ Jianfeng Mao*b and Guoxin Sun ${ }^{\mathrm{a}}$
}

Non-noble metal-based catalysts with efficient catalytic activities for the oxygen evolution reaction (OER) and oxygen reduction reaction (ORR) are critical for energy conversion devices, including fuel cells and metal-air batteries. In this work, novel hafnium phosphide-reduced graphene oxide nanosheets (HfPrGO NS) and hafnium disulfide-reduced graphene oxide nanosheets ( $\mathrm{HfS}_{2}-\mathrm{rGO} N \mathrm{NS}$ ) were synthesized and investigated as bifunctional electrocatalysts for OER and ORR. The prepared HfP-rGO NS and $\mathrm{HfS}_{2}{ }^{-}$ rGO NS catalysts showed nanosheet structures, where the HfP or $\mathrm{HfS}_{2}$ nanosheet was closely packed with $\mathrm{rGO}$. A unique methodology was adopted to lodge the non-metal oxide catalytic sheets (i.e., HfP and $\mathrm{HfS}_{2}$ ) over the $\mathrm{rGO}$ sheets, which positioned the oxide layer on the catalytic sheet surface for instant oxygen evolution. Low intensity X-ray diffraction patterns and Raman spectra confirmed the sheet-like structure of HfP-rGO NS and $\mathrm{HfS}_{2}-\mathrm{rGO}$ NS. Scanning electron microscope mapping images revealed that all elements (i.e., $\mathrm{Hf}, \mathrm{P}, \mathrm{C}$ and $\mathrm{O}$ for $\mathrm{HfP}-\mathrm{rGO} \mathrm{NS}$ and $\mathrm{Hf}, \mathrm{S}, \mathrm{C}$ and $\mathrm{O}$ for $\mathrm{HfS}_{2}-\mathrm{rGO} \mathrm{NS}$ ) were equally distributed in the synthesized heteroatomic nanosheets. Moreover, both the HfP-rGO NS and $\mathrm{HfS}_{2}-\mathrm{rGO}$ NS demonstrated excellent durability for both ORR and OER. This outperforms the most state-of-the-art non-precious-metal-based bifunctional catalysts, which is attributed to the synergistic effect of rGO and $\mathrm{Hf}$-based catalysts. The different ORR and OER reaction potentials in $\mathrm{HfP}-\mathrm{rGO} \mathrm{NS}$ and $\mathrm{HfS}_{2}-\mathrm{rGO} \mathrm{NS}$ likely result from the influence of $\mathrm{HfP}$ and $\mathrm{HfS}_{2}$.

\section{Introduction}

Renewable electrochemical energy conversion technologies, such as fuel cells and metal-air batteries, are in high demand in today's society in order to reduce environmental pollution and to act as a replacement for fossil fuels. ${ }^{1-5}$ In general, fuel cells are primary energy devices that generate electricity via a redox reaction between the fuel anode and oxygen cathode. Reversible fuel cells are similar secondary energy devices that can reduce oxygen $\left(\mathrm{O}_{2}\right)$ and, in reverse, produce $\mathrm{O}_{2}$. Therefore, the oxygen evolution reaction (OER; $4 \mathrm{OH}^{-} \rightarrow \mathrm{O}_{2}+2 \mathrm{H}_{2} \mathrm{O}+4 \mathrm{e}^{-}$) and the oxygen reduction reaction $\left(\mathrm{ORR} ; \mathrm{O}_{2}+2 \mathrm{H}_{2} \mathrm{O}+4 \mathrm{e}^{-} \rightarrow 4 \mathrm{OH}^{-}\right)$are

${ }^{a}$ School of Chemistry and Chemical Engineering, University of Jinan, 336 West Road of Nanxinzhuang, Jinan, Shandong, 250022,China.E-mail:chm_huangtz@ujn.edu.cn ${ }^{b}$ Institute for Superconducting \& Electronic Materials, Australian Institute for Innovative Materials, University of Wollongong, Wollongong, NSW, 2522, Australia. E-mail:jmao@uow.edu.au

$\dagger$ Electronic supplementary information (ESI) available: XPS spectra, high-resolution oxygen spectra and elemental mappings of HfP-rGO NS and $\mathrm{HfS}_{2}$-rGO NS. OER bode plots, $\mathrm{N}_{2}$-saturated ORR CVs, ORR LSV and ORR bode plots of HfP-rGO, $\mathrm{HfS}_{2}$-rGO and Pt/C catalysts. See DOI: 10.1039/c8ra09598a the two vital electrochemical reactions at the oxygen cathode that drive these devices. ${ }^{6-8}$ However, the slow and sluggish kinetics of these oxygen reactions at the air cathode result in high overpotential and poor oxygen adsorption, which severely limit the overall efficiency of the working cells. ${ }^{9-16}$ Therefore, designing bifunctional electrocatalysts for both OER and ORR is highly desirable for reversible fuel cell and metal-oxygen battery systems.

Until now, many research articles have showcased electrocatalysts for either ORR or OER; but only a few reports on both ORR and OER have been published. Noble metals, such as Pt, $\mathrm{Ru}, \mathrm{Rh}, \mathrm{Pd}$ and $\mathrm{Au}$, for example, have been reported as promising and highly efficient electrocatalysts. Among them, Pt is the most well-known active electrocatalyst for the ORR, but is limited in terms of the OER because of the formation of Pt oxide on the catalyst surface. ${ }^{14,17}$ Due to the high cost of noble metals, transition-metal-based compounds such as metal oxides, metal carbides, metal nitrides, metal sulfides and metal phosphides have been utilized as alternative catalysts because they are abundant, cheap, electrochemically active and chemically stable. ${ }^{17-20}$ Among them, metal oxides such as $\mathrm{Co}_{3} \mathrm{O}_{4}$, NiO and 
$\mathrm{MnO}_{2}$ have been considered as the most promising alternatives due to the easy transfer of $\mathrm{O}^{2-}$ from the metal-oxygen bonds. Nevertheless, reported metal oxide catalysts still suffer from high overpotential, low catalytic activity and poor cycling stability., ${ }^{9,14}$ Meanwhile, most of the metal oxides and hydroxides have been used only for the OER. ${ }^{21}$ Compared with transition metal oxides, transition metal sulfides usually possess better electronic conductivity. Considering their cost, performance stability and safety issues, the metal sulfides are very attractive for electrochemical applications. ${ }^{22-24}$ Similarly, the metal phosphides are also capable of excellent catalytic performance and durability, and also have higher electrical conductivity than oxides. In particular, metal phosphides are used for OERs by the virtue of their proton-acceptor characteristics which allows them to form molecules easily (i.e., oxygen, hydrogen $e t c.)^{25,26}$

In addition, metal-based electrocatalysts and carbon-based materials have also been employed as bifunctional active catalysts due to their good intrinsic OER reactivity and corrosion resistivity at high oxidation potentials. Meanwhile, doping heteroatoms to nanocarbon materials can lead to the favorable formation of $\mathrm{OH}^{-}$via the direct $4 \mathrm{e}^{-}$pathway, and could significantly boost ORR activity. Oxygen reduction catalysis via doped N, S, P, B or I on dual-atom-doped carbon nanotubes (CNTs) and/or graphene materials has been studied broadly. ${ }^{14}$ This is because the heteroatoms can tune the electronic properties of $\mathrm{C}$ and offer more active sites. ${ }^{6,27}$ In particular, reduced graphene oxide (rGO) attracting the interest due to its few-layer or multi-layer atomic carbon sheets, as well as the presence of both $\mathrm{C}$ and $\mathrm{O}$ (heteroatoms), which make them more favorable for electrochemical reactions. Moreover, the catalytic performance of graphenebased materials could be further improved by incorporating transitional metals. For example, electrocatalysts such as $\mathrm{Co}_{3} \mathrm{O}_{4} /$ $\mathrm{N}$-rmGO and $\mathrm{Co}_{4} \mathrm{~N} / \mathrm{rGO}$ have been discussed as highly active catalysts for the ORR because of their well-assorted heteroatoms. ${ }^{8,28}$ The combination of rGO sheets with metallic compounds (i.e., heteroatom doping) can improve the electronic conductivity and increase the surface area, and hence make the electrocatalyst highly active due to the synergistic effects. ${ }^{14,29,30}$ Similarly, many active heterogeneous catalysts, such as FeP@NPC, ${ }^{31}$ FeP@PNC-800, ${ }^{32} \mathrm{Fe}_{x} \mathrm{P} / \mathrm{NPCS},{ }^{25}$ rGO-Co-Pi, ${ }^{33}$ N-CG$\mathrm{CoO},{ }^{1} \mathrm{~V}(\mathrm{C}, \mathrm{N}),{ }^{34} \mathrm{~N}-\mathrm{Co}_{9} \mathrm{~S}_{8} / \mathrm{G}^{35}$ and FeS NS, ${ }^{36}$ have been reported for the ORR/OER. However, the need remains to achieve highperformance bifunctional electrocatalysts in bulk applications for energy conversion devices based on oxygen electrocatalysis.

In this work, hafnium disulfide-reduced graphene oxide nanosheets ( $\mathrm{HfS}_{2}$-rGO NS) and hafnium phosphide-reduced graphene oxide nanosheets (HfP-rGO NS) were electrochemically tested for the OER and ORR in $0.1 \mathrm{M} \mathrm{KOH}$ electrolyte solution, and their intrinsic bifunctional behavior for oxygen reactions was demonstrated. In addition, instead of anchoring the catalytic nanoparticles on the rGO sheets, this work combines the catalytic (Hf) nanosheets with rGO sheets for better stability and high synergistic effect during the oxygen reactions. Interestingly, both $\mathrm{HfP}-\mathrm{rGO} \mathrm{NS}$ and $\mathrm{HfS}_{2}-\mathrm{rGO} \mathrm{NS}$ are competitive with each other in different aspects of the OER and ORR electrochemical measurements.

\section{Experimental methods}

\section{Preparation of HfP-rGO and $\mathrm{HfS}_{2}$-rGO nanosheets}

HfP-rGO NS were prepared by the following procedure. $150 \mathrm{mg}$ of red phosphorous was dissolved in $50 \mathrm{ml}$ of ethylene glycol under constant stirring. After fine dissolution, $150 \mathrm{mg}$ of hafnium tetrachloride $\left(\mathrm{HfCl}_{4}\right)$ was added pinch-by-pinch into the stirring solution. The solution mixture was ultra-sonicated for $1 \mathrm{~h}$ and moved to a Teflon flask to be heated at $200{ }^{\circ} \mathrm{C}$ in the microwave oven for $15 \mathrm{~h}$. After heating, the solution mixture was cooled naturally, and then the dark red-colored HfP slurry was collected and washed with ethanol. ${ }^{37}$ The obtained HfP slurry was dissolved in $50 \mathrm{ml}$ of ultra-pure (UP) water and sonicated for $2 \mathrm{~h}$. In parallel, $17 \mathrm{ml}$ of GO ink $\left(6 \mathrm{mg} \mathrm{ml}^{-1}\right)$ was dissolved in $30 \mathrm{ml}$ of UP water and sonicated for $2 \mathrm{~h}$. The GO ink was prepared by the modified-Hummers' method. ${ }^{8}$ After $2 \mathrm{~h}$, the sonicated GO solution was added dropwise into the sonicating HfP solution. After that, the physically mixed HfP-GO nanosheet solution was sonicated for a further $30 \mathrm{~min}$ and then transferred into a round-bottom flask. Next, $3 \mathrm{ml}$ of hydrazine hydrate $\left(\mathrm{H}_{4} \mathrm{~N}_{2} \cdot \mathrm{H}_{2} \mathrm{O}\right)$ was dropped into the HfP-GO solution, and then the round-bottom flask was refluxed for $3 \mathrm{~h}$ at $120^{\circ} \mathrm{C}$ in an oil bath. After the chemical reduction, dark red-colored HfPrGO NS slurry was washed with ethanol and UP water separately. Then the slurry was completely dried at $80{ }^{\circ} \mathrm{C}$ and the HfP-rGO NS catalyst powder was collected and finely ground.

The above procedure was also followed to prepare $\mathrm{HfS}_{2}$-rGO $\mathrm{NS}$, except that $300 \mathrm{mg}$ of $\mathrm{Na}_{2} \mathrm{~S}$ crystals and $150 \mathrm{mg}$ of $\mathrm{HfCl}_{4}$ were used in the $24 \mathrm{~h}$ solvothermal treatment at $200{ }^{\circ} \mathrm{C} .{ }^{38} \mathrm{In}$ this process, black-colored $\mathrm{HfS}_{2}$-rGO NS powder was obtained.

\section{Material characterization}

Crystals of the prepared $\mathrm{HfS}_{2}$-rGO NS and HfP-rGO NS catalysts were analyzed by powder X-ray diffraction (XRD) using a Bruker D8 advanced diffractometer with $\mathrm{Cu} K \alpha$ radiation $(\lambda=1.5418 \AA$ A $)$. Raman spectra of $\mathrm{HfS}_{2}$-rGO NS and HfP-rGO NS were recorded using a Renishaw inVia spectrometer with $532 \mathrm{~nm}$ laser excitation. Morphologies of the $\mathrm{HfS}_{2}$-rGO NS and HfP-rGO NS catalysts were captured by scanning electron microscopy (SEM), and the elements in the catalysts were distinguished by elemental mapping techniques using a Hitachi (S-4800) SEM. The in-depth morphology of the prepared catalysts was further examined by transmission electron microscopy (TEM) and selected area electron diffraction tests using a Tecnai $(20 \mathrm{U}$ TWIN) TEM. The elemental composition and the binding energies of the $\mathrm{HfS}_{2}$-rGO NS and HfP-rGO NS were detected using a Thermo Fisher Scientific ESCALAB 250Xi X-ray photoelectron spectrometer (XPS) with $\mathrm{Al} \mathrm{K} \alpha$ radiation.

\section{Electrochemical measurements}

All the ORR and OER electrochemical measurements of the prepared $\mathrm{HfS}_{2}$-rGO NS and HfP-rGO NS catalysts were carried out using a CHI 760D electrochemical workstation. For ORR measurements, cyclic voltammetry (CV), linear sweeping voltammetry (LSV), the construction of Tafel plots, electrochemical impedance spectroscopy and current-time $(i-t)$ 
chronoamperometric tests were conducted using a threeelectrode half-cell set-up (glassy carbon disk-working electrode, graphite rod-counter electrode and $\mathrm{Ag} / \mathrm{AgCl}$-reference electrode) in $0.1 \mathrm{M} \mathrm{KOH}$ electrolyte solution. For $\mathrm{N}_{2}$ and $\mathrm{O}_{2}$ saturated electrolytes, $\mathrm{N}_{2}$ and $\mathrm{O}_{2}$ gases were bubbled into the $0.1 \mathrm{M} \mathrm{KOH}$ electrolyte for $1 \mathrm{~h}$ each. $\mathrm{CV}$ curves were recorded in $\mathrm{N}_{2}$-saturated electrolyte at a sweeping rate of $20 \mathrm{mV} \mathrm{s}^{-1}$. A forward scanning potential range from $0.2 \mathrm{~V}$ to $-0.8 \mathrm{~V}(1.16 \mathrm{~V}$ to $0.16 \mathrm{~V}$ in reversible hydrogen electrode [RHE]) was maintained for the ORR. Afterwards, the $\mathrm{O}_{2}$ was passed into the electrolyte, and then CV, LSV, Tafel, AC impedance and amperometric $i-t$ curve tests were conducted. Similarly, a rotating ring-disk electrode (RRDE) and 3A electrode were used for the rotating disk electrode (RDE) and RRDE tests, respectively, in $\mathrm{N}_{2}$ - and $\mathrm{O}_{2}$ saturated 0.1 M KOH electrolyte. In the RDE tests, CV curves were first recorded in $\mathrm{N}_{2}$-saturated $0.1 \mathrm{M} \mathrm{KOH}$, then the $\mathrm{CV}$ and LSV were conducted in $\mathrm{O}_{2}$-saturated electrolyte. The LSV curves were recorded at a sweeping rate of $5 \mathrm{mV} \mathrm{s}^{-1}$ with a disk rotating speed in the range of 400, 625, 900, 1225, 1600 and $2025 \mathrm{rpm}$. In the RRDE tests, the LSV test at $5 \mathrm{mV} \mathrm{s}^{-1}$ was recorded at a rotating speed of $1600 \mathrm{rpm}$ using the RRDE.

For OER measurements, the $\mathrm{O}_{2}$ gas was bubbled in $0.1 \mathrm{M}$ $\mathrm{KOH}$ for $1 \mathrm{~h}$ and all the electrochemical tests were carried out with the RDE electrode with $1600 \mathrm{rpm}$ continuous rotation. At first, the LSV test was conducted at $5 \mathrm{mV} \mathrm{s}^{-1}$ in the reverse scan potential ranges from $0 \mathrm{~V}$ to $1.2 \mathrm{~V}(0.96 \mathrm{~V}$ to $2.16 \mathrm{~V}$ in $\mathrm{RHE})$, then Tafel, AC impedance and amperometric $i-t$ curve tests were conducted for OER. In order to prepare the catalyst layer on the electrode, $3 \mathrm{mg}$ of either $\mathrm{HfS}_{2}$-rGO NS or HfP-rGO NS was mixed with $30 \mu \mathrm{l}$ of Nafion and $270 \mu \mathrm{l}$ of UP water $(18.25 \mathrm{M} \Omega$ ) and sonicated for $1 \mathrm{~h}$ to obtain the catalyst ink. Then, $5 \mu \mathrm{l}(10 \mu \mathrm{l}$ for the RRDE electrode) of the catalyst ink was pipetted onto the working electrode. All working electrodes were polished and washed with ethanol and deionized water before the catalyst was loaded.

\section{Results and discussion}

\section{Structural characterization}

XRD patterns for the prepared $\mathrm{HfS}_{2}$-rGO NS and HfP-rGO NS catalysts are shown in Fig. 1a, revealing that sharp intense peaks were not found due to the non-crystalline, sheet-like structures. For $\mathrm{HfS}_{2}-\mathrm{rGO} \mathrm{NS}$, a broad peak ranging from $20^{\circ}$ to $40^{\circ}$ and comprising $\mathrm{HfS}_{2}$ and $\mathrm{C}$ is seen, in which the high intensity is due to the presence of $\mathrm{C}$. The presence of $\mathrm{HfS}_{2}$ is confirmed by tiny peaks at $28.2^{\circ}(100), 32.3^{\circ}(101), 42.2^{\circ}(102)$, $55.4^{\circ}(103)$ and $61.3^{\circ}(201)$, which are indexed to JCPDS file no. 28-0444. Tiny diffraction peaks of $\mathrm{C}$ were detected at $31.2^{\circ}$, $26.6^{\circ}, 45.6^{\circ}, 66.1^{\circ}$ and $75.3^{\circ}$ (JCDPS no. 46-0943). The HfP-rGO NS have the highest intense peak due to C (005) at $25.3^{\circ}$. After C (005), the peak starts to fall gradually until $40^{\circ}$, after which HfP (101) is seen at $29.1^{\circ} . \mathrm{C}(102)$ at $42.8^{\circ}$ results in a sharp peak, which confirms the confinement of the carbon atomic sheet from GO. Furthermore, HfP (110) and HfP (114) are identified by small peaks at $50.7^{\circ}$ and $58.2^{\circ}$, respectively (JCPDS no. 65-3506). At $78^{\circ}$, a sharp peak for $\mathrm{C}(110)$ is seen that is indexed to JCPDS no. 26-1077. The XRD patterns of the $\mathrm{HfS}_{2}$-rGO nanosheets
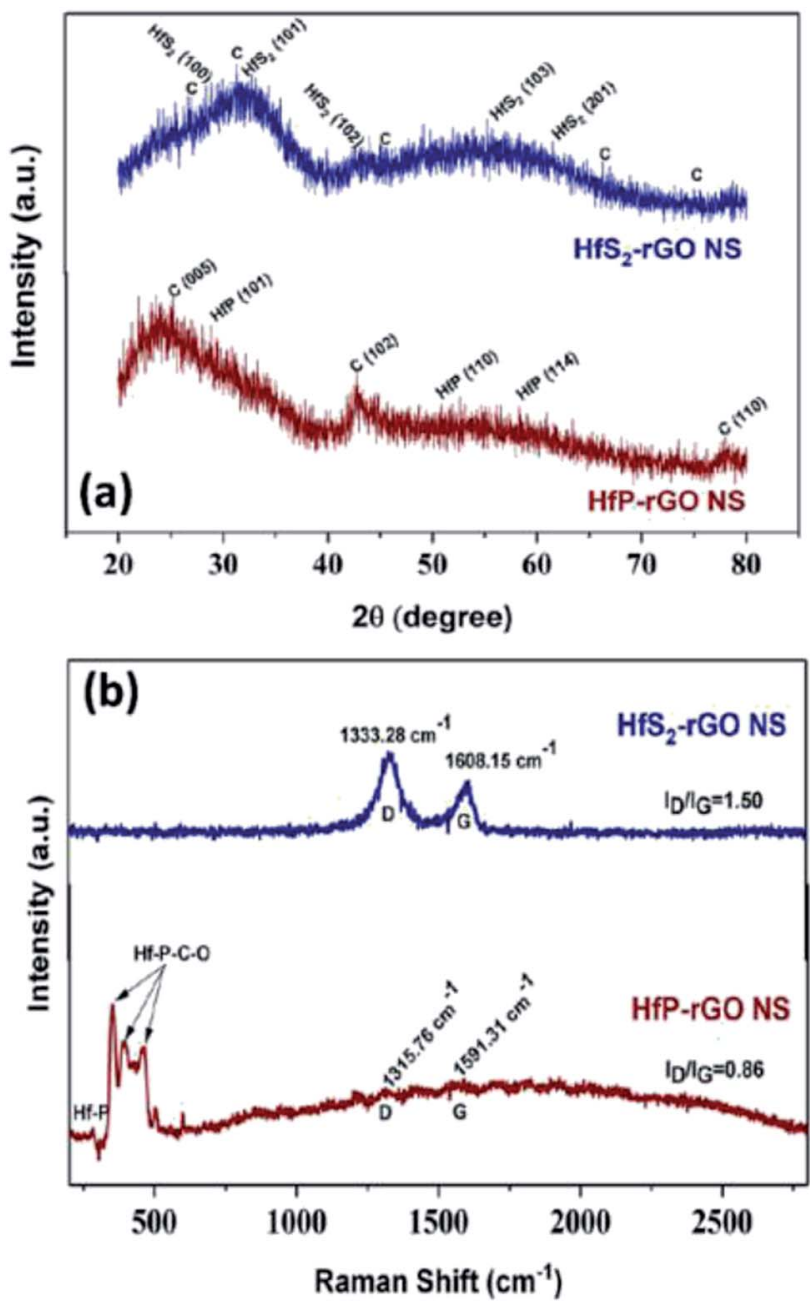

Fig. 1 (a) X-ray diffraction patterns and (b) Raman spectra of $\mathrm{HfS}_{2}-\mathrm{rGO}$ nanosheets and HfP-rGO nanosheets.

shows a broad hump, which can be mainly attributed to sulfide $\left(\mathrm{HfS}_{2}\right)$ and rGO. Also, the weak $\mathrm{C}$ peaks indicate the nanosheets with thick rGO layers. For HfP-rGO nanosheets, the oxideinfluenced humps are not found; instead, sharp C peaks are seen due to the thin carbon sheets. Fig. 1b shows the Raman spectra of HfP-rGO NS and $\mathrm{HfS}_{2}$-rGO NS. The D and G peaks of the $\mathrm{HfS}_{2}$-rGO nanosheets are positioned at $1333.28 \mathrm{~cm}^{-1}$ and $1608.15 \mathrm{~cm}^{-1}$, respectively. The intensity ratio of the $\mathrm{D}$ and $\mathrm{G}$ bands (i.e., $I_{\mathrm{D}} / I_{\mathrm{G}}$ for $\mathrm{HfS}_{2}$-rGO NS) is $1.50 . I_{\mathrm{D}} / I_{\mathrm{G}} \gg 1$ indicates that the intensity of the $\mathrm{D}$ band was higher than that of the $\mathrm{G}$ band, which means that more structural defects exist in the rGO. ${ }^{8,39}$ The Raman spectra of HfP-rGO NS show a peak at $285.15 \mathrm{~cm}^{-1}$, which can be attributed to Hf-P. Strong intense peaks at $358.41 \mathrm{~cm}^{-1}, 392.77 \mathrm{~cm}^{-1}$ and $467.55 \mathrm{~cm}^{-1}$ are attributed to the effect of heteroatomic elements such as $\mathrm{C}$ and $\mathrm{O}$, which influence the phosphide ( $\mathrm{Hf}-\mathrm{P}-\mathrm{C}-\mathrm{O}$ ), while forming the homogeneous HfP-rGO nanosheets. ${ }^{40}$ The $\mathrm{D}$ and $\mathrm{G}$ peaks for HfP-rGO NS are obtained at $1315.76 \mathrm{~cm}^{-1}$ and $1591.31 \mathrm{~cm}^{-1}$, respectively. Here, $I_{\mathrm{D}} / I_{\mathrm{G}}=0.86$ (i.e., minimal structural defects) occurred on the $\mathrm{C}$ atoms. The Raman spectra reveal that HfPrGO NS is atomically thin and consists of low structure defects in the carbons. ${ }^{41}$ 

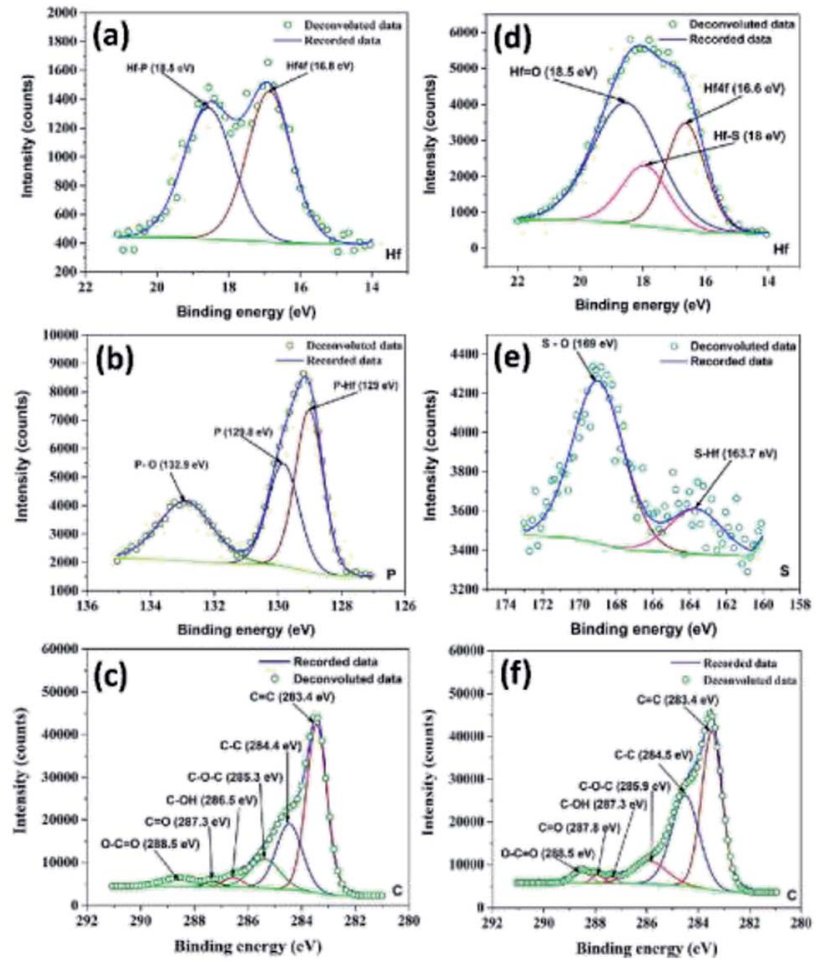

Fig. 2 High-resolution XPS spectra of (a) hafnium, (b) phosphorus and (c) carbon for HfP-rGO nanosheets. High-resolution XPS spectra of (d) hafnium, (e) sulfur and (f) carbon for $\mathrm{HfS}_{2}-\mathrm{rGO}$ nanosheets.

Meanwhile, XPS spectra of the as-prepared HfP-rGO NS and $\mathrm{HfS}_{2}$-rGO NS catalysts are shown in Fig. S1a and $\mathrm{c}$ in the ESI. $\dagger$ The high-resolution XPS of Hf for HfP-rGO NS is shown in Fig. 2a. The Hf $4 \mathrm{f}$ peak at $16.8 \mathrm{eV}$ and $\mathrm{Hf}-\mathrm{P}$ at $18.5 \mathrm{eV}$ form twin peaks for Hf in HfP-rGO NS. The equivalent phosphide peak (Hf-P) rising next to $\mathrm{Hf} 4 \mathrm{f}$ shows the presence of $\mathrm{Hf}$ and phosphide formation in the prepared HfP-rGO NS. Similarly, highresolution $\mathrm{Hf}$ XPS of $\mathrm{HfS}_{2}-\mathrm{rGO} \mathrm{NS}$ is shown in Fig. 2d, in which $\mathrm{Hf}$ 4f is visible at $16.6 \mathrm{eV}$ along with a sulfide peak (Hf-S) at $18 \mathrm{eV}$ and an oxide peak $(\mathrm{Hf}=\mathrm{O})$ at $18.5 \mathrm{eV}$, combining to form a single peak. Here, the intensity of the $\mathrm{Hf}=\mathrm{O}$ peak is higher than that of $\mathrm{Hf} 4 \mathrm{f}$ and $\mathrm{Hf}-\mathrm{S}$, which should be increased by the reaction of rGO. However, in the HfP-rGO NS, the oxide effects are substantially controlled by elemental phosphorus. Fig. 2b shows the high-resolution XPS of phosphorus for HfPrGO NS, in which the elevated peak at $129 \mathrm{eV}$ corresponds to $\mathrm{P}-\mathrm{Hf}$, and an associated peak at $129.8 \mathrm{eV}$ is attributed to elemental phosphorus. A small peak at $132.9 \mathrm{eV}$ may be attributed to P-O. In parallel, the high-resolution XPS for sulfur in $\mathrm{HfS}_{2}$-rGO is shown in Fig. 2e. A small peak at $163.7 \mathrm{eV}$ shows the presence of $\mathrm{HfS}_{2}$, whereas the S-O peak is found at $169 \mathrm{eV}$. The comparison of the sulfur XPS for $\mathrm{HfS}_{2}$-rGO NS and phosphorus XPS spectra for HfP-rGO NS clearly demonstrates that the oxide effect from rGO has a minimal influence on the HfP, whereas $\mathrm{HfS}_{2}$ failed to control. The C XPS for HfP-rGO NS and $\mathrm{HfS}_{2}$-rGO NS are shown in Fig. 2c and $\mathrm{f}$, respectively. The $\mathrm{C}=\mathrm{C}$ peak at $283.4 \mathrm{eV}$ in both the HfP-rGO and $\mathrm{HfS}_{2}$-rGO NS catalysts are attributed to the $\mathrm{sp}^{2}$ carbon bond $(\mathrm{C}=\mathrm{C})$ of rGO. Next, the $\mathrm{C}-\mathrm{C}$ peak is visible at $284.4 \mathrm{eV}$ for $\mathrm{HfP}-\mathrm{rGO} \mathrm{NS}$ and $284.5 \mathrm{eV}$ for
$\mathrm{HfS}_{2}$-rGO NS. Conversely, the $\mathrm{C}-\mathrm{C}$ peak $\left(\mathrm{sp}^{3}\right.$ bond) intensity is higher in $\mathrm{HfS}_{2}$-rGO NS due to the presence of more structural defects on $\mathrm{C}$. The $\mathrm{C}-\mathrm{O}-\mathrm{C}$ peak is visible at $285.3 \mathrm{eV}$ in HfP-rGO $\mathrm{NS}$ and at $285.9 \mathrm{eV}$ in $\mathrm{HfS}_{2}$-rGO NS. Small peaks with binding energies centered at $286.5 \mathrm{eV}$ and $287.3 \mathrm{eV}$ in HfP-rGO NS may be attributed to $\mathrm{C}-\mathrm{OH}$ and $\mathrm{C}=\mathrm{O}$ bonds, but these were found at $287.3 \mathrm{eV}(\mathrm{C}-\mathrm{OH})$ and $287.8 \mathrm{eV}(\mathrm{C}=\mathrm{O})$ in $\mathrm{HfS}_{2}$-rGO NS. The $\mathrm{O}-\mathrm{C}=\mathrm{O}$ peak is seen at $288.5 \mathrm{eV}$ in both $\mathrm{HfP}-\mathrm{rGO} \mathrm{NS}$ and $\mathrm{HfS}_{2}-$ rGO NS. ${ }^{42}$ The increased intensity of the $\mathrm{O}-\mathrm{C}=\mathrm{O}$ peak in $\mathrm{HfS}_{2}$ rGO NS is likely caused by the high oxides stacked in the rGO nanosheets. The $\mathrm{O} 1 \mathrm{~s}$ peak at $530.8 \mathrm{eV}$ is obtained in HfP-rGO $\mathrm{NS}$, next to that, $\mathrm{O}=\mathrm{C}$ at $532.3 \mathrm{eV}$ is visible easily due to the strong $\mathrm{C}$ and $\mathrm{O}$ bonding in the rGO sheets. For $\mathrm{HfS}_{2}$-rGO NS, O $1 \mathrm{~s}$ is obtained at $531.6 \mathrm{eV}$ and a small $\mathrm{O}=\mathrm{C} \mathrm{peak}^{43}$ is raised at $533.4 \mathrm{eV}$ within the $\mathrm{O} 1 \mathrm{~s}$ peak, which reveals that the bonding between the $\mathrm{C}$ and $\mathrm{O}$ in the rGO nanosheet is hindered. ${ }^{4}$

The SEM image of HfP-rGO NS in Fig. 3a shows the sheet-like structures, and the corresponding elemental mappings of $\mathrm{Hf}, \mathrm{P}$, $\mathrm{C}$ and $\mathrm{O}$ are shown in Fig. S2a-d, $\dagger$ respectively. Fig. $\mathrm{S} 2 \dagger$ demonstrates that all elements (Hf, P, C and O) are homogeneously distributed over the sheet-like structures. Similarly, the SEM image of $\mathrm{HfS}_{2}$-rGO NS is shown in Fig. 3d, which reveals a sheet-like surface. The corresponding elemental mappings of $\mathrm{Hf}, \mathrm{S}, \mathrm{C}$ and $\mathrm{O}$ are shown in Fig. S3a-d $\dagger$ respectively. Similar to HfP-rGO NS, all elements (Hf, S, C and O) are distributed equally on the nanosheets, although the occurrence of $\mathrm{O}$ is illuminated more strongly on the elemental mapping images. Fig. $3 \mathrm{~b}$ and e are the TEM images of HfP-rGO NS and $\mathrm{HfS}_{2}$-rGO NS. It can be clearly seen that the sheet-like structures are captured and no particles are detected. The high-resolution TEM images of HfPrGO NS and $\mathrm{HfS}_{2}$-rGO NS are shown in Fig. 3c and f, respectively, in which thin sheets collide at their edges. The thickness of the edges in HfP-rGO NS are found to be $0.9 \mathrm{~nm}$ and $1.6 \mathrm{~nm}$, respectively, whereas the edge thicknesses of the $\mathrm{HfS}_{2}$-rGO nanosheets are $2.7 \mathrm{~nm}$ and $3.6 \mathrm{~nm}$.

\section{Electrochemical analysis}

The oxygen reduction behavior of the prepared HfP-rGO NS, $\mathrm{HfS}_{2}$-rGO NS and commercial Pt/C catalysts are shown as CVs in Fig. 4a. The pre-determined $\mathrm{N}_{2}$-saturated CVs of HfP-rGO NS, $\mathrm{HfS}_{2}$-rGO NS and Pt/C catalysts are shown in Fig. S4. $\dagger$ HfP-rGO NS catalyst started its oxygen reduction at $0.97 \mathrm{~V} v s$. RHE $\left(E_{\text {on }}\right)$, whereas the onset potential $\left(E_{\text {on }}\right)$ of $\mathrm{HfS}_{2}$-rGO NS was obtained at $0.95 \mathrm{~V}$. As usual, $\mathrm{Pt} / \mathrm{C}(20 \mathrm{wt} \%)(1.05 \mathrm{~V})$ is dominating on the $E_{\text {on }}$ of ORR. Highly reactive P towards O ensured that the HfPrGO NS delivered the maximum current density of $1.163 \mathrm{~mA}$ $\mathrm{cm}^{-2}$ at $0.79 \mathrm{~V}\left(E_{\text {peak }}\right)$, which is higher than that of Pt/C $(0.850$ $\left.\mathrm{mA} \mathrm{cm}{ }^{-2}\right)$ and $\mathrm{HfS}_{2}$-rGO NS $\left(0.612 \mathrm{~mA} \mathrm{~cm}^{-2}\right)$. The peak potential $\left(E_{\text {peak }}\right)$ of $\mathrm{HfS}_{2}$-rGO NS is $0.82 \mathrm{~V}$, which is higher than that of the prepared phosphide-based (HfP-rGO NS) catalyst. In general, the metal-phosphide electrocatalysts exhibit oxidation peaks during the ORR CV measurements in the alkaline testing environment, which confirms an oxidation peak by HfP-rGO NS catalyst at $0.89 \mathrm{~V} v$ s. RHE. ${ }^{25}$ Although Pt/C attained the highest ORR potentials (i.e., $E_{\text {on }}$ potential at $1.05 \mathrm{~V}$ and $E_{\text {peak }}$ at $0.95 \mathrm{~V}$ ), its active ORR current density $\left(0.850 \mathrm{~mA} \mathrm{~cm}^{-2}\right)$ is $27 \%$ lower 

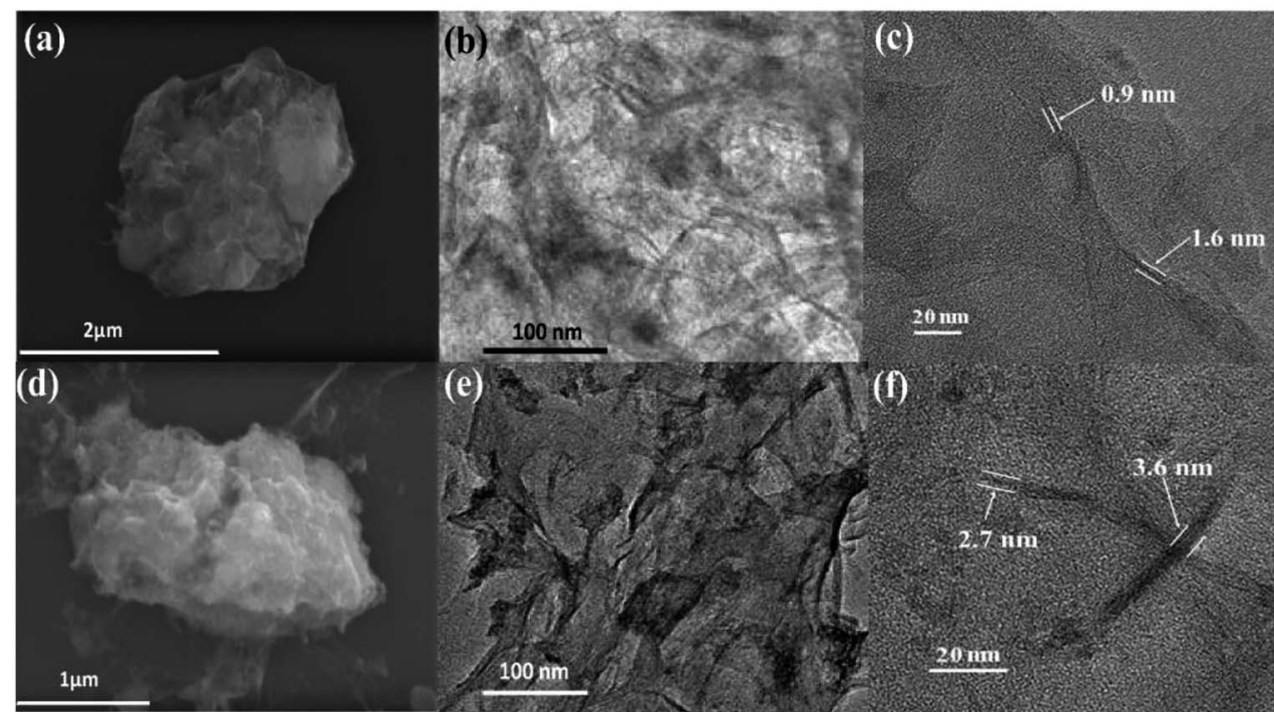

(e)

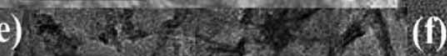

(1)

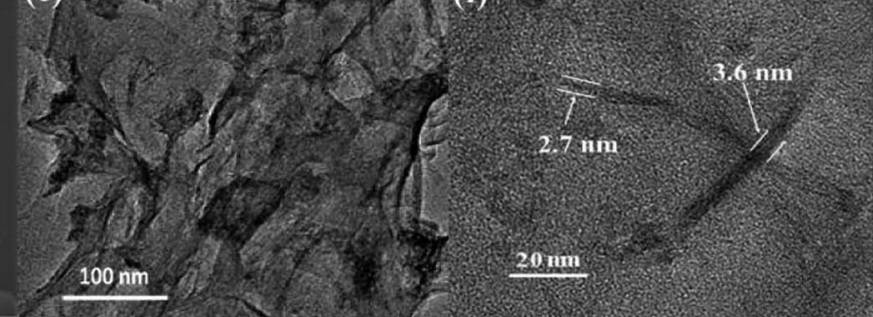

Fig. 3 (a) SEM, (b) TEM and (c) HRTEM image of HfP-rGO nanosheets. (d) SEM, (e) TEM and (f) HRTEM image of Hff ${ }_{2}-r G O$ nanosheets.
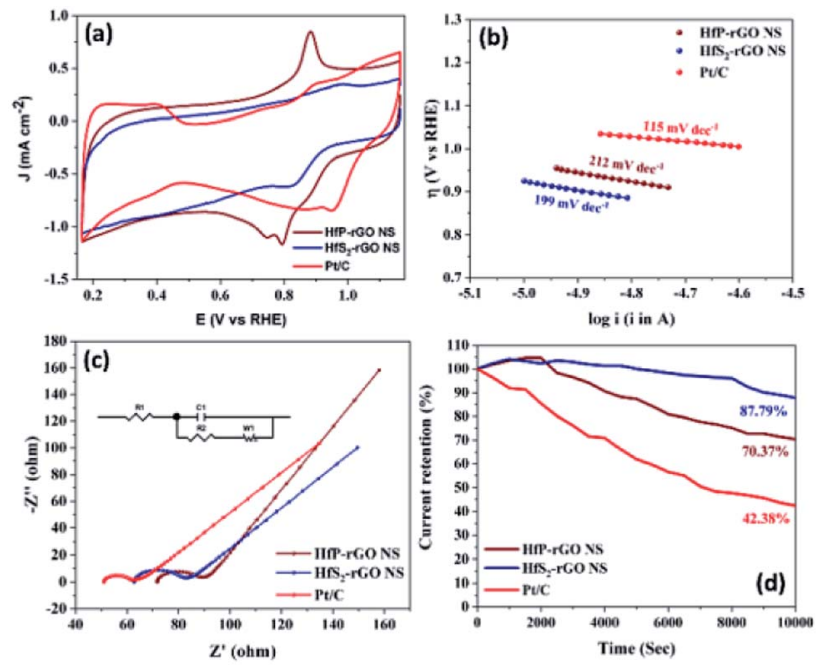

Fig. 4 ORR electrochemical measurements of $\mathrm{HfP}-\mathrm{rGO}$ and $\mathrm{HfS}_{2}-$ rGO nanosheets: (a) cyclic voltammograms in $\mathrm{O}_{2}$ saturated $0.1 \mathrm{M} \mathrm{KOH}$ solution at a sweeping rate of $20 \mathrm{mV} \mathrm{s}^{-1}$, (b) Tafel plots, (c) Nyquist plots with corresponding equivalent circuits (inset) and (d) amperometric $i-t$ curve studies.

than that of HfP-rGO nanosheets. Linear sweep voltammograms are shown in Fig. S5, $\dagger$ in that HfP-rGO NS delivered a greater ORR current than that of $\mathrm{Pt} / \mathrm{C}$ and $\mathrm{HfS}_{2}$-rGO NS. ORR Tafel plots are shown in Fig. 4b, in which both HfP-rGO NS and $\mathrm{HfS}_{2}$-rGO NS had a lower overpotential ( $\eta$ ) than that of Pt/C. Due to the impact of ORR, Pt/C achieved a lower Tafel slope value $\left(115 \mathrm{mV} \mathrm{dec}{ }^{-1}\right)^{45}$ than HfP-rGO NS $\left(212 \mathrm{mV} \mathrm{dec}^{-1}\right)$ and $\mathrm{HfS}_{2}-$ rGO NS (199 $\left.\mathrm{mV} \mathrm{dec}^{-1}\right)$. The current exchange density and the electron transfer coefficient can be determined using Tafel's eqn (1): ${ }^{8}$

$$
\eta=a+b \log i=-\frac{2.303 R T}{a n F} \log i_{0}+\frac{2.303 R T}{a n F} \log i
$$

where $R$ is the universal gas constant, $T$ is the absolute temperature in K, $F$ is Faraday's constant, $b$ is the Tafel slope, $a$ is the charge transfer coefficient, $n$ is the electron transfer coefficient and $i_{0}$ is the exchange current density. The electron transfer coefficients of $\mathrm{HfP}-\mathrm{rGO} \mathrm{NS}$ and $\mathrm{HfS}_{2}$-rGO NS were found to be 0.56 and 0.59 , respectively. Incredibly, the exchange current density of $\mathrm{HfS}_{2}$-rGO NS $\left(142.85 \times 10^{-3} \mathrm{~A} \mathrm{~cm}^{-2}\right)$ and HfPrGO NS $\left(16.24 \times 10^{-3} \mathrm{~A} \mathrm{~cm}^{-2}\right)$ are higher than that of $\mathrm{Pt} / \mathrm{C}(2.8$ $\times 10^{-7} \mathrm{~A} \mathrm{~cm}^{-2}$ ) catalyst. The catalytic activities of HfP-rGO NS, $\mathrm{HfS}_{2}-\mathrm{rGO} \mathrm{NS}$ and $\mathrm{Pt} / \mathrm{C}$ were further investigated by Nyquist plots, as shown in Fig. 4c. The inserted circuit model is the corresponding equivalent circuit. The ohmic resistance $R_{1}$ of HfP-rGO NS, $\mathrm{HfS}_{2}$-rGO NS and Pt/C are 71.76, 62.76 and $51 \Omega$, respectively. The capacitive layer $\left(C_{1}\right)$ between the catalyst surface and electrolyte is found to be very thin in HfP-rGO NS $(0.00095 \mathrm{~F})$ compared with that of $\mathrm{HfS}_{2}$-rGO NS $(0.00038 \mathrm{~F})$ and $\mathrm{Pt} / \mathrm{C}(0.00005 \mathrm{~F})$; i.e., phosphides have a thin active layer, which is two-fold bigger in the sulfides. $R_{2}$, the reaction resistance between the catalyst surface and electrolyte, in multi-layered oxide-enriched $\mathrm{HfS}_{2}$-rGO NS shows the highest resistance of $16 \Omega\left(R_{2}\right)$. In contrast, $\mathrm{Pt} / \mathrm{C}$ nanoparticles achieved the lowest $R_{2}$ $(9 \Omega)$, whereas the median HfP-rGO obtained was $14 \Omega$, confirming the surface conductivity of phosphides is lower than that of sulfides. The ORR is concerned with the Warburg resistance $\left(W_{1}\right)$, in which both the HfP-rGO NS and $\mathrm{HfS}_{2}$-rGO NS catalysts achieved $16 \Omega$ due to wide-spread catalytic centers, whereas the active $\mathrm{Pt} / \mathrm{C}$ achieved $17 \Omega$ for its hidden active spots. Fig. S6 $†$ shows the Bode plots, in which HfP-rGO displays the lowest frequency due to high electron life availability. The ORR catalytic stabilities of HfP-rGO NS, $\mathrm{HfS}_{2}-\mathrm{rGO} \mathrm{NS}$ and Pt/C catalysts are shown in Fig. 4d for 10000 s. Due to rapid degradation of $\mathrm{Pt}$, the $\mathrm{Pt} / \mathrm{C}$ is the main loser among the prepared catalysts, which reached $42.38 \%$ of its current at the end of the stability test. The sheet-structured HfP-rGO NS and $\mathrm{HfS}_{2}$-rGO NS strengthened the heteroatomic catalytic sites and maintained $70.37 \%$ and $87.79 \%$, respectively. The early rise of the ORR 

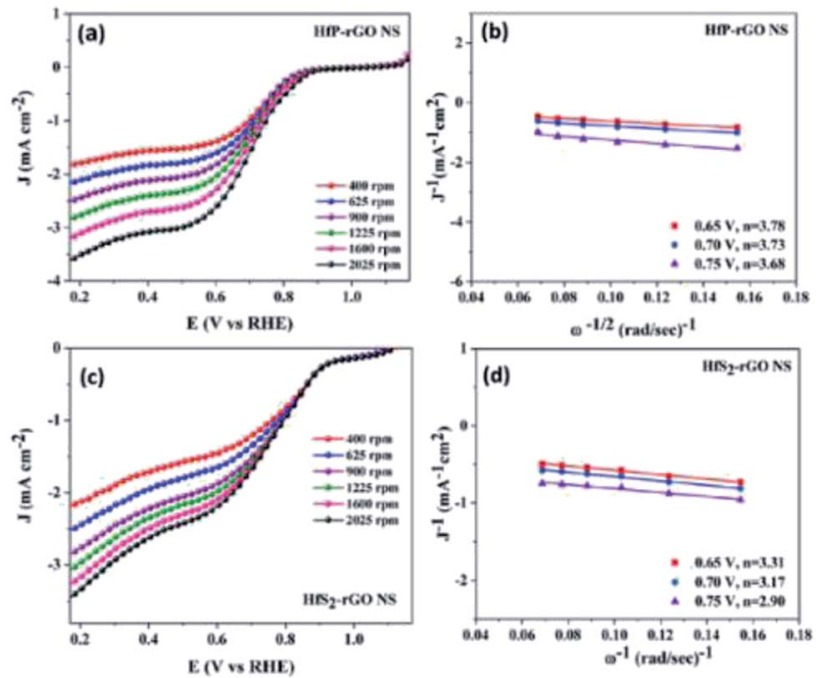

Fig. 5 (a) Rotating disk electrode voltammograms and (b) $K-L$ plots of $\mathrm{HfP}-\mathrm{rGO}$ NS. (c) Rotating disk electrode voltammograms and (d) $\mathrm{K}-\mathrm{L}$ plots of $\mathrm{HfS}_{2}-\mathrm{rGO}$ NS.

current percentage is attributed to the initial reduction reaction of the available catalytic sites on the sheet towards the oxygen. The improved stability of $\mathrm{HfS}_{2}$-rGO was mainly due to the oxideenriched nanosheets, whereas phosphide active centers reacted quickly towards $\mathrm{O}$, causing further current loss to the HfP-rGO NS catalyst.

The electrocatalytic activity and electron kinetics of the ORR for the prepared HfP-rGO NS and $\mathrm{HfS}_{2}$-rGO NS catalysts were further investigated using an RDE. The RDE voltammetry curves of $\mathrm{HfP}-\mathrm{rGO} \mathrm{NS}$ and $\mathrm{HfS}_{2}$-rGO $\mathrm{NS}$ at various rotation rates are shown in Fig. 5a and c, respectively. Similar to CV, RDE onset potentials of the HfP-rGO nanosheet are higher than that of $\mathrm{HfS}_{2}$-rGO NS. Surprisingly, the diffusion current is well controlled by phosphides in HfP-rGO NS to fully reduce the $\mathrm{O}_{2}$ and to yield minimum hydrogen peroxide. Fig. $5 \mathrm{~b}$ and $\mathrm{d}$ are the corresponding Koutechy-Levich (K-L) plots of HfP-rGO NS and $\mathrm{HfS}_{2}$-rGO NS. The following Koutechy-Levich eqn (2) is used to determine the kinetics of electrons during the ORR:

$$
\frac{1}{J}=\frac{1}{J_{\mathrm{L}}}+\frac{1}{J_{\mathrm{K}}}=\frac{1}{B \omega^{1 / 2}}+\frac{1}{J_{\mathrm{K}}}
$$

where $J$ is the measured current density, $J_{\mathrm{K}}$ is the kinetic current density and $J_{\mathrm{L}}$ is the diffusion limiting current density. The angular frequency $\omega$ can be found using $\omega=2 \pi N / 60$, where $N$ is the rotation rate of the working electrode. ${ }^{8}$ From the KoutechyLevich equation, $B$, the slope of the $\mathrm{K}-\mathrm{L}$ line can be written as following eqn (3):

$$
B=0.62 n F C_{0}\left(D_{0}\right)^{2 / 3} \vartheta^{-1 / 6}
$$

where $n$ is the electron transfer number, $F$ is the Faraday constant, $D_{0}$ is the diffusion coefficient of $\mathrm{O}_{2}$ in the electrolyte, $\vartheta$ is the kinematic viscosity and $C_{0}$ is the bulk concentration of the $\mathrm{O}_{2}$ in the solution. ${ }^{8}$ Using the above equation, the electron transfer numbers $(n)$ of HfP-rGO NS and $\mathrm{HfS}_{2}$-rGO NS are calculated at the active potential region. The $n$ values in the $\mathrm{K}-\mathrm{L}$ plots indicate that both HfP-rGO NS and $\mathrm{HfS}_{2}$-rGO NS follow the direct $4 \mathrm{e}^{-}$reduction process. RRDE voltammograms of HfP-rGO NS and $\mathrm{HfS}_{2}$-rGO NS are shown in Fig. 6a. RRDE results once again confirm that phosphides of HfP-rGO NS are capable of delivering a higher current than the sulfides of the $\mathrm{HfS}_{2}$-rGO NS during the ORR. The electron transfer number and the percentage of yielded hydrogen peroxide during the RRDE tests for HfP-rGO NS and $\mathrm{HfS}_{2}$-rGO NS are shown in Fig. $6 \mathrm{~b}$ and c, respectively. The electron transfer number $n$ and $\mathrm{H}_{2} \mathrm{O}_{2} \%$ can be calculated using the below eqn (4) and (5):

$$
\begin{gathered}
n=\frac{4 \times I_{\mathrm{d}}}{\left(I_{\mathrm{d}}+\frac{I_{\mathrm{r}}}{\mathrm{N}}\right)}, \\
\mathrm{H}_{2} \mathrm{O}_{2} \%=200 \times \frac{\frac{I_{\mathrm{r}}}{\mathrm{N}}}{I_{\mathrm{d}}+\frac{I_{\mathrm{r}}}{\mathrm{N}}},
\end{gathered}
$$

where $I_{\mathrm{d}}$ is the disk current, $I_{\mathrm{r}}$ is the ring current and $N$ is the geometrical current collection coefficient (for $\mathrm{Pt}, N=0.39$ ). In the active ORR region, HfP-rGO NS had ' $n$ ' ranges between 3.75 and 3.9, but $\mathrm{HfS}_{2}$-rGO NS transferred electrons between 3.25 and 3.5. The $\mathrm{H}_{2} \mathrm{O}_{2} \%$ data reveal that HfP-rGO NS yielded just 5$10 \%$ of hydrogen peroxide. However, $\mathrm{HfS}_{2}$-rGO NS produced $30-$ $40 \%$ of hydrogen peroxide production, which is nearly fourtimes higher than that of HfP-rGO NS. The oxygen evolution catalytic activities of the prepared HfP-rGO NS, $\mathrm{HfS}_{2}$-rGO NS and commercial $\mathrm{Pt} / \mathrm{C}$ catalysts are illustrated in the polarization
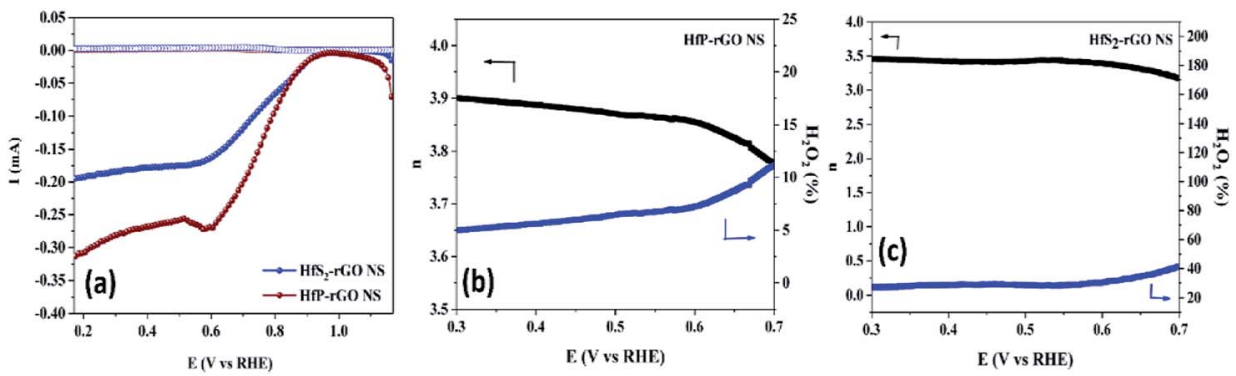

Fig. 6 (a) Rotating ring-disk electrode voltammograms of $\mathrm{HfP}-\mathrm{rGO} N \mathrm{NS}$ and $\mathrm{HfS}_{2}-\mathrm{rGO}$ NS. Electron transfer number ' $n$ ' and $\mathrm{H}_{2} \mathrm{O}_{2} \%$ production for (b) HfP-rGO NS and (c) $\mathrm{HfS}_{2}$-rGO NS. 

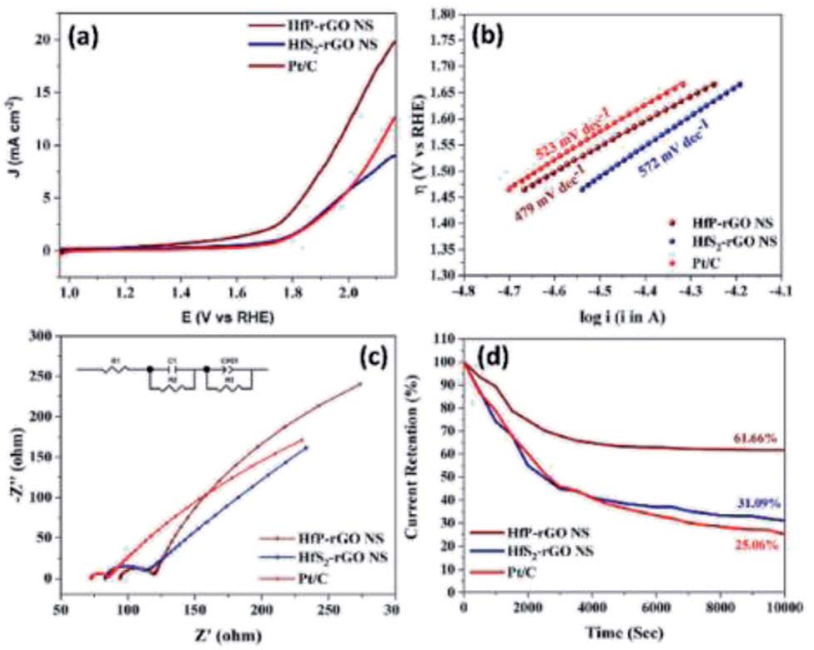

Fig. 7 (a) OER polarization curves in $0.1 \mathrm{M} \mathrm{KOH}$ solution at a sweeping rate of $5 \mathrm{mV} \mathrm{s}^{-1}$ (rotation: $1600 \mathrm{rpm}$ ), (b) Tafel plots, (c) Nyquist plots with corresponding equivalent circuit (inset) and (d) amperometric $i-t$ test results of $\mathrm{HfP}-\mathrm{rGO}$ and $\mathrm{HfS}_{2}-\mathrm{rGO}$ nanosheets.

test, and the results are shown in Fig. 7a. HfP-rGO NS showed the highest OER current, which reached up to $19 \mathrm{~mA} \mathrm{~cm}^{-2}$ by the positive ion-accepting nature of phosphide to form oxygen. Both $\mathrm{HfS}_{2}$-rGO NS and Pt/C began oxygen evolution at $1.78 \mathrm{~V} v s$. RHE, but HfP-rGO NS started the OER at $1.72 \mathrm{~V}$ with a higher current density.

To gain further insights into the highly active phosphidebased OER catalysts, HfP-rGO NS have attained the lowest potential at $1.96 \mathrm{~V}$, which is lower than that of $\mathrm{Pt} / \mathrm{C}(2.12 \mathrm{~V})$ and $\mathrm{HfS}_{2}$-rGO NS (beyond $2.12 \mathrm{~V}$ ) for the constant current density measurement of $10 \mathrm{~mA} \mathrm{~cm}{ }^{-2}$. The OER Tafel plots of HfP-rGO $\mathrm{NS}, \mathrm{HfS}_{2}$-rGO NS and Pt/C are shown in Fig. 7b. In accordance with the lower OER potentials, HfP-rGO NS achieved the lowest Tafel slope value (479 $\mathrm{mV} \mathrm{dec}^{-1}$ ) compared with $\mathrm{HfS}_{2}-\mathrm{rGO}$ $\left(572 \mathrm{mV} \mathrm{dec}^{-1}\right)$ and Pt/C $\left(523 \mathrm{mV} \mathrm{dec}^{-1}\right)$. The Nyquist plots of HfP-rGO NS, $\mathrm{HfS}_{2}$-rGO NS and Pt/C are shown in Fig. 7c. The modulated equivalent circuit is inserted. $R_{1}$ is the ohmic resistance and $R_{2}$ is the reaction resistance between the electrolyte and the catalyst surface. The formed capacitive layer $\left(C_{1}\right)$ between the catalyst surface and the electrolyte signify a small semi-circle in the Nyquist plots. Due to the greater formation of $\mathrm{O}_{2}$, the capacitance $\left(C_{1}\right)$ of $\mathrm{HfS}_{2}$-rGO NS $(4538 \mu \mathrm{F})$ is higher than that of Pt/C (545 $\mu \mathrm{F})$ and HfP-rGO NS $(357 \mu \mathrm{F})$. Next to the semicircle, all catalysts have a linear curve rise that actually represents the OER resistance $\left(R_{3}\right)$. The efficient OER of HfP-inducted rGO catalyst is affirmed again by the least $R_{3}$ value of $850 \Omega$. The $\mathrm{HfS}_{2}$-influenced catalyst ( $\mathrm{HfS}_{2}$-rGO NS) offers three-fold higher resistance (2900 $\Omega$ ). Incredibly, HfP-rGO NS again dominates over the commercial Pt/C catalyst (1028 $\Omega$ ). The produced oxygen molecules on the catalyst surface imply the presence of a capacitive layer (CPE), in which HfP-rGO NS (0.0460) has a higher OER active layer than $\mathrm{HfS}_{2}$-rGO NS (0.1096) and Pt/C (0.1181). The OER Bode plots are shown in Fig. S7, $\dagger$ in which $\mathrm{Pt} / \mathrm{C}$ has a maximum frequency, which means a shorter electron life, ${ }^{34}$ whereas HfP-rGO NS and $\mathrm{HfS}_{2}$-rGO NS have a better

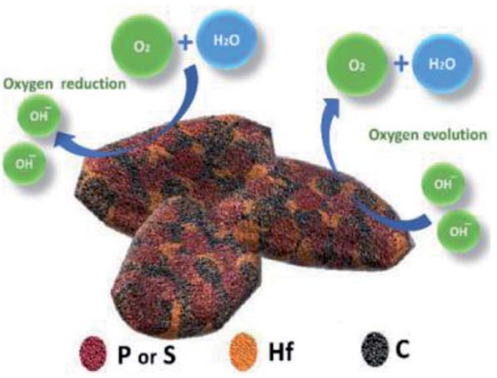

Fig. 8 Structure and elemental arrangements of $\mathrm{HfP}-\mathrm{rGO} / \mathrm{HfS}_{2}-\mathrm{rGO}$ nanosheets, and oxygen reduction and oxygen evolution reactions.

electron life due to the stable sheet-like catalyst structure. The OER catalytic stabilities of $\mathrm{HfP}-\mathrm{rGO} \mathrm{NS}, \mathrm{HfS}_{2}$-rGO NS and Pt/C catalysts for $10000 \mathrm{~s}$ are shown in Fig. 7d. Similar to the OER polarization curves, the performances of $\mathrm{HfS}_{2}$-rGO and $\mathrm{Pt} / \mathrm{C}$ are quite similar. The nanosheet-structured $\mathrm{HfS}_{2}-\mathrm{rGO}$ showed better stability than $\mathrm{Pt} / \mathrm{C}$ and persisted up to $31.09 \%$ of its OER current; whereas Pt/C has drained to $25.06 \%$ at the end. The active site-enriched thin HfP-rGO nanosheets were maintained at $61.66 \%$ and emerged as a stable OER catalyst.

Fig. 8 shows the structure of the prepared HfP-rGO and $\mathrm{HfS}_{2}$-rGO nanosheets and the bifunctional oxygen reactions. Both the HfP-rGO NS and $\mathrm{HfS}_{2}$-rGO NS showed superior catalytic activities compared with most catalysts for ORR and OER. For OER, at the current density of $5 \mathrm{~mA} \mathrm{~cm}{ }^{-2}$, HfP-rGO NS achieved the potential at $1.82 \mathrm{~V}$ vs. RHE, which is lower than that of other catalysts such as $\mathrm{IrO}_{2}(1.85 \mathrm{~V}),{ }^{9} \mathrm{RuO}_{2}(1.85$ $\mathrm{V}),{ }^{10} \mathrm{Pt} / \mathrm{C}(1.92 \mathrm{~V})$ and $\mathrm{HfS}_{2}$-rGO NS (1.92 V). As discussed earlier, the proton-acceptor property of phosphide-based HfPrGO NS has an outstanding catalytic activity for $\mathrm{O}_{2}$ evolution. For ORR, the onset potential of HfP-rGO NS ( $0.97 \mathrm{~V} v s$. RHE) is lower than that of Pt/C (1.05 V vs. RHE), but higher than that of $\mathrm{HfS}_{2}$-rGO NS (0.95 V vs. RHE), N/Fe-co-doped G $\left.(0.87 \mathrm{~V})\right)^{46}$ FeP@NPC (0.9 V), ${ }^{31} \mathrm{FeNiS}_{2} \mathrm{NS}(0.79 \mathrm{~V}),{ }^{36} \mathrm{Ni}_{9} \mathrm{~S}_{8} \mathrm{NR}(0.68 \mathrm{~V}),{ }^{36}$ FeS NS (0.32 V), ${ }^{36} \mathrm{FeP} @ P N C-800(0.76 \mathrm{~V}),{ }^{32} \mathrm{Fe}_{x} \mathrm{P} / \mathrm{NPCS}(0.9 \mathrm{~V}),{ }^{25}$ rGO-Co-Pi $(0.91 \mathrm{~V}),{ }^{33} \mathrm{Co}_{4} \mathrm{~N} / \mathrm{rGO}(0.87 \mathrm{~V})^{8}$ and N-CG-CoO (0.9 V) ${ }^{1}$ catalysts. Similarly, the ORR peak current density $\left(J_{\text {peak }}\right)$ of HfP-rGO NS $\left(1.16 \mathrm{~mA} \mathrm{~cm}^{-2}\right)$ is superior to $\mathrm{Pt} / \mathrm{C}\left(0.85 \mathrm{~mA} \mathrm{~cm}^{-2}\right)$, $\mathrm{HfS}_{2}$-rGO NS (0.61 mA cm $\left.{ }^{-2}\right)$, FeP@PNC-800 $\left(0.8 \mathrm{~mA} \mathrm{~cm}{ }^{-2}\right),{ }^{32}$ rGO-Co-Pi $\left(0.35 \mathrm{~mA} \mathrm{~cm}^{-2}\right)^{33}$ and $\mathrm{V}(\mathrm{C}, \mathrm{N})\left(0.13 \mathrm{~mA} \mathrm{~cm}^{-2}\right)^{34}$ catalysts. Furthermore, the peak potential of ORR of HfP-rGO NS (0.79 V vs. RHE) is higher than that of FeP@PNC-800 (0.58 $\mathrm{V}),{ }^{32} \mathrm{Co}_{9} \mathrm{~S}_{8} / \mathrm{G}(0.69 \mathrm{~V})^{35}$ and $\mathrm{N}-\mathrm{Co}_{9} \mathrm{~S}_{8} / \mathrm{G}(0.7 \mathrm{~V}) .{ }^{1}$ Thus, the measured electrochemical data and the discussions confirm that phosphide-based HfP-rGO nanosheets are predominant towards $\mathrm{O}_{2}$ evolution. For ORR, the hafnium phosphides delivered higher reaction currents, but sulfide-enriched $\mathrm{HfS}_{2}$ rGO NS maintained better ORR catalytic stability and higher ORR potentials at the peak to provide more energy for $\mathrm{O}_{2}$ reduction. The different electrocatalytic activities in HfP-rGO NS and $\mathrm{HfS}_{2}$-rGO NS are due to the different anions. Thus, the prepared nanosheet-like HfP and hafnium disulfide catalysts tuned by rGO sheets achieved the oxygen reactions efficiently by a synergistic effect. 


\section{Conclusions}

The influence of phosphides and sulfides in the prepared HfPrGO NS and $\mathrm{HfS}_{2}$-rGO NS catalysts for ORR and OER were investigated. Due to the proton-acceptor ability, phosphideenriched HfP-rGO NS has played a phenomenal role towards $\mathrm{O}_{2}$ evolution. The OER potential of HfP-rGO NS is lower than that of $\mathrm{HfS}_{2}$-rGO NS, Pt/C, $\mathrm{RuO}_{2}$ and $\mathrm{IrO}_{2}$, which indicates the superior catalytic activity and potential as an alternative to noble OER catalysts. During the ORR, $\mathrm{HfS}_{2}$-rGO NS exhibited higher catalytic stability and better Tafel slope activity; however, hafnium phosphides achieved higher ORR current density than that of $\mathrm{HfS}_{2}-\mathrm{rGO} \mathrm{NS}$ and $\mathrm{Pt} / \mathrm{C}$. Also, the ORR onset potential of HfP-rGO NS is just $80 \mathrm{mV}$ lower than that of the Pt/C. In particular, both the HfP-rGO NS and $\mathrm{HfS}_{2}$-rGO NS catalysts have achieved remarkable $16 \Omega$ of Warburg diffusion resistance during the ORR. This is the first report concerning Hf-based bifunctional oxygen catalysts. The preparation technique and electrochemical characteristics of these prepared HfP-rGO NS and $\mathrm{HfS}_{2}$-rGO NS catalysts might accelerate understanding with respect to the design and development of robust oxygen catalysts.

\section{Conflicts of interest}

There are no conflicts to declare.

\section{Acknowledgements}

This work was financially supported by the Shandong Provincial Natural Science Foundation, China (No. ZR2018MB036, ZR2017LB005), Science Development Project of Shandong Province (No. 2017GGX40115), National Natural Science Foundation of China (Grant No. 51302022). Mayilvel Dinesh Meganathan is grateful for the financial support of the China Scholarship Council for International Doctoral Students in China (No. 2015DFH586).

\section{Notes and references}

1 S. Mao, Z. Wen, T. Huang, Y. Hou and J. Chen, Energy Environ. Sci., 2014, 7, 609-616.

2 J. Yang, T. Fujigaya and N. Nakashima, Sci. Rep., 2017, 7, 45384.

3 F. Cheng and J. Chen, Chem. Soc. Rev., 2012, 41, 2172-2192. 4 V. Ramani, Electrochem. Soc. Interface, 2006, 15(1), 41-44.

5 G. Sandstede, E. J. Cairns, V. S. Bagotsky and K. Wiesener, History of low temperature fuel cells, John Wiley \& Sons, 2010.

6 J. Zhang, Z. Zhao, Z. Xia and L. Dai, Nat. Nanotechnol., 2015, 10, 444-452.

7 R. Jadhav, H. A. Bandal, A. H. Tamboli and H. Kim, J. Energy Chem., 2017, 26, 695-702.

8 M. D. Meganathan, S. Mao, T. Huang and G. Sun, J. Mater. Chem. A, 2017, 5, 2972-2980.

9 Y. Dou, T. Liao, Z. Ma, D. Tian, Q. Liu, F. Xiao, Z. Sun, J. Ho Kim and S. Xue Dou, Nano Energy, 2016, 30, 267-275.
10 J. Wang, Y. Fu, Y. Xu, J. Wu, J.-H. Tian and R. Yang, Int. J. Hydrogen Energy, 2016, 41, 8847-8854.

11 Z. Wang, M. Li, L. Fan, J. Han and Y. Xiong, Appl. Surf. Sci., 2017, 401, 89-99.

12 D. Sun, Y. Shen, W. Zhang, L. Yu, Z. Yi, W. Yin, D. Wang, Y. Huang, J. Wang, D. Wang and J. B. Goodenough, J. Am. Chem. Soc., 2014, 136, 8941-8946.

13 Y.-C. Lu, Z. Xu, H. A. Gasteiger, S. Chen, K. Hamad-Schifferli and Y. Shao-Horn, J. Am. Chem. Soc., 2010, 132, 12170-12171.

14 J. Fu, Z. P. Cano, M. G. Park, A. Yu, M. Fowler and Z. Chen, Adv. Mater., 2017, 29, 1604685.

15 K. Kinoshita, Electrochemical Oxygen Technology, John Wiley \& Sons, 1992, vol. 30.

16 Y. Sun, C. Li and G. Shi, J. Mater. Chem., 2012, 22, 1281012816.

17 S. Guo, S. Zhang and S. Sun, Angew. Chem., Int. Ed., 2013, 52, 8526-8544.

18 S. Dou, L. Tao, J. Huo, S. Wang and L. Dai, Energy Environ. Sci., 2016, 9, 1320-1326.

19 C.-H. Lai, M.-Y. Lu and L.-J. Chen, J. Mater. Chem., 2012, 22, 19-30.

20 M. Chen, J. Liu, W. Zhou, J. Lin and Z. Shen, Sci. Rep., 2015, 5, 10389.

21 X. Xu, F. Song and X. Hu, Nat. Commun., 2016, 7, 12324.

22 Y. Wu, C. Wadia, W. Ma, B. Sadtler and A. P. Alivisatos, Nano Lett., 2008, 8, 2551-2555.

23 T.-L. Li, Y.-L. Lee and H. Teng, J. Mater. Chem., 2011, 21, 5089.

24 M. J. Bierman and S. Jin, Energy Environ. Sci., 2009, 2, 1050. 25 K. Hu, Z. Xiao, Y. Cheng, D. Yan, R. Chen, J. Huo and S. Wang, Electrochim. Acta, 2017, 254, 280-286.

26 Y. Wang, B. Kong, D. Zhao, H. Wang and C. Selomulya, Nano Today, 2017, 15, 2655.

27 M. Seredych, K. László, E. Rodríguez-Castellón and T. J. Bandosz, J. Energy Chem., 2016, 25, 236-245.

28 Y. Liang, Y. Li, H. Wang, J. Zhou, J. Wang, T. Regier and H. Dai, Nat. Mater., 2011, 10, 780-786.

29 Y. Shao, F. Ding, J. Xiao, J. Zhang, W. Xu, S. Park, J.-G. Zhang, Y. Wang and J. Liu, Adv. Funct. Mater., 2013, 23, 987-1004.

30 H. Wang and H. Dai, Chem. Soc. Rev., 2013, 42, 3088-3113.

31 R. Zhang, C. Zhang and W. Chen, J. Mater. Chem. A, 2016, 4, 18723-18729.

32 X. Xu, C. Shi, R. Chen and T. Chen, RSC Adv., 2017, 7, 2226322269.

33 Z. G. Zhao, J. Zhang, Y. Yuan, H. Lv, Y. Tian, D. Wu and Q. W. Li, Sci. Rep., 2013, 3, 2263.

34 J. Yu, X. Gao, G. Chen and X. Yuan, Int. J. Hydrogen Energy, 2016, 41, 4150-4158.

35 S. Dou, L. Tao, J. Huo, S. Wang and L. Dai, Energy Environ. Sci., 2016, 9, 1320-1326.

36 J. Jiang, S. Lu, H. Gao, X. Zhang and H.-Q. Yu, Nano Energy, 2016, 27, 526-534.

37 Y.-M. Hu, M.-C. Liu, Y.-X. Hu, Q.-Q. Yang, L.-B. Kong and L. Kang, Electrochim. Acta, 2016, 215, 114-125.

38 Q. R. Hu, S. L. Wang, Y. Zhang and W. H. Tang, J. Alloys Compd., 2010, 491, 707-711.

39 A. Kaniyoor and S. Ramaprabhu, AIP Adv., 2012, 2, 032183. 
40 A. M. Elshahawy, C. Guan, X. Li, H. Zhang, Y. Hu, H. Wu, S. J. Pennycook and J. Wang, Nano Energy, 2017, 39, 162-171. 41 Q. Hu, M. Hirai, R. K. Joshi and A. Kumar, J. Phys. D: Appl. Phys., 2009, 42, 025301.

42 H. Tao, C. Yan, A. W. Robertson, Y. Gao, J. Ding, Y. Zhang, T. Ma and Z. Sun, Chem. Commun., 2017, 53, 873-876.

43 Z. Sun, J. Masa, P. Weide, S. M. Fairclough, A. W. Robertson, P. Ebbinghaus, J. H. Warner, S. C. E. Tsang, M. Muhler and W. Schuhmann, J. Mater. Chem. A, 2015, 3, 15444.
44 XPS Simplified Online, accessed Apr 2018.

45 C. Wen, X. Gao, T. Huang, X. Wu, L. Xu, J. Yu, H. Zhang, Z. Zhang, J. Han and H. Ren, Int. J. Hydrogen Energy, 2016, 41, 11099-11107.

46 H. Tao, Y. Gao, N. Talreja, F. Guo, J. Texter, C. Yan and Z. Sun, J. Mater. Chem. A, 2015, 5, 7257-7284. 